\title{
CONTABILIDAD DE GESTIÓN: UN ESTUDIO EN REVISTAS DE BRASIL, ESPAÑA Y DE LENGUA ESPAÑOLA
}

\author{
MANAGEMENT ACCOUNTING: A STUDY IN BRAZILIAN JOURNALS AND \\ SPANISH LANGUAGE
}

\author{
Rogério João Lunkes ${ }^{a}$; Vicente Mateo Ripoll-Feliu ${ }^{b}$; Fabricia Silva da Rosa ${ }^{c}$ \\ ${ }^{a}$ Professor Adjunto IV da Universidade Federal de Santa Catarina, UFSC; \\ Doutorado em Engenharia de Produção pela Universidade Federal de Santa Catarina, UFSC; \\ Florianópolis,SC-Brasil;E-mail: rogeriolunkes@hotmail.com \\ ${ }^{b}$ Professor Titular de Universidad do Universitat Valencia, Espanha; \\ Doutorado pelo Universitat Valencia, Espanha; \\ Valencia, - Espanha; E-mail: Vicente.Ripoll@uv.es \\ Pesquisadora do Departamento de Ciências Contábeis na Universidade Federal de Santa Catarina, UFSC, \\ Doutora em Engenharia de produção pela UFSC com doutorado sanduiche em em Contabilidade na Universitat de Valencia; \\ Florianópolis, SC-Brasil; E-mail: fabriciasrosa@hotmail.com

\section{Resumen}

En las últimas décadas, la contabilidad de gestión ha experimentado importantes cambios con la inclusión de nuevos temas y métodos de investigación. Una manera de darnos cuenta y entender el nivel de desarrollo y las características de esta evolución, es el estudio de sus publicaciones en revistas. Así el objetivo del presente trabajo es identificar y analizar los temas y métodos de investigación aplicada en los estudios de contabilidad de gestión en España, Brasil y en países de Lengua Española. Con este fin, se seleccionaron, entre 2001 y 2010, en primer lugar, siete revistas contables españolas que figuran en la base de datos IN-RECS (Índice de Impacto de Revistas Españolas de Ciencias Sociales) en donde hemos detectado 421 artículos, en segundo lugar se han seleccionado seis revistas brasileñas que están evaluadas por CAPES (Coordinación de Perfeccionamiento de la Educación Superior), en donde se han detectado 245 artículos, y en tercer lugar se han seleccionado cinco revistas de lengua española que figuran en la base de datos Latindex, en donde hemos seleccionado 52 artículos. Los resultados muestran que los trabajos en contabilidad de gestión no han ocupado un lugar destacado en las publicaciones analizadas en el ámbito contable. Entre los temas destacados se encuentran la planificación y control, con énfasis en el elemento de medición y evaluación del desempeño, y en el caso de Brasil, los artículos predominantes se basan en el control organizacional. El desarrollo de los estudios se produce, en gran medida, con la revisión de la literatura y los métodos de estudio de casos.

Palabras clave: Contabilidad de Gestión, Temas, Métodos, Literatura.

\begin{abstract}
In recent decades, management accounting has undergone major changes with the inclusion of new topics and research methods. One way to realize and understand the level of development and characteristics of this evolution is the study of his publications in journals. Thus, the objective of this work is to identify and analyze issues and research methods applied in studies of management accounting in Spain, Brazil and Spanish Language. To this end, we selected between 2001 and 2010, first seven Spanish accounting journals contained in the database INRECS where we have identified 421 articles, second Instead we have selected six journals are evaluated by Brazilian CAPES, where they have identified 245 articles, and third place have selected five Spanish-language journals listed in the database Latindex where we have selected 52 articles. The results show that jobs in management accounting have not figured prominently in the papers reviewed in the accounting field. Among the issues highlighted include the planning and control, with emphasis on the element of performance measurement and evaluation, and in the case of Brazil, the predominant items are based on organizational control. The development of the studies produced largely with the literature review and case study methods.
\end{abstract}

Keywords: Management Accounting, Topics, Methods. Bibliographic. 


\section{INTRODUCCIÓN}

En las últimas décadas, la contabilidad de gestión ha experimentado un cambio considerable, con la inclusión de nuevos temas de investigación (Johnson y Kaplan, 1987; Cooper, 1987; Kaplan, 1983, Hiromoto, 1988; Bromwich, 1990; Dent 1990; Yong y Selton, 1991, Kaplan y Norton, 1992, Shank y Govindarajan, 1993; Simons, 1995), y especialmente los estudios con perspectivas multidisciplinares (Baiman, 1982, Cooper 1983; Covaleski et al 1996; Macintoch y Scapens, 1990, Kaplan y Norton, 2001).

También en este periodo se ha consolidado la disciplina de contabilidad de gestión en las universidades como una ciencia social. Esto aumentó la credibilidad y el estado de este campo del conocimiento (Baldvinsdottir; Norreklit y Mitchell, 2010), lo cual ha llevado a un aumento de investigaciones en contabilidad de gestión y en la teoría o teorías que la sustentan (Ittner y Larcker, 2001 y 2002, Zimmerman, 2001; Hopwood, 2002; Lukka y Mouritsen, de 2002, Luft y Shields, 2002; Chapman, Hopwood y Shields, 2007, Malmi y Granlund, 2009; Vaivio y Sirén, 2010; Modell, 2010; Lukka, 2010; Merchant, 2010), centradas en los preceptos básicos, las teorías centrales, los enfoques (decisión o control), y los métodos de exploración (descriptivo o normativo).

Es importante analizar la coherencia de los métodos de investigación, debido a la existencia de un número limitado de revistas con un enfoque específico, con la finalidad de abrir nuevos debates que promuevan el progreso científico de la contabilidad de gestión.

Varios estudios han sido desarrollados con el fin de evaluar la producción científica de contabilidad de gestión en nivel internacional (Carvalho et al 2010; Nascimento, Junqueira y Martins, 2010; Cardoso, Pereira y Guerreiro, 2007; Mendonca, Riccio y Sakata, 2009; Beuren, Schlindwein y Pasqual, 2007; Carter, Maloni, y Carr, 2009; Hesford y Potter, 2010; Alcouffe, Berland y Levant, 2008; Groot y Valderrama-García, 2006; Valacich, et al 2006; Tahai y Meyer, 1999; Engwall, 1998; White, 1996, Kirkpatrick y Locke, 1992). En España se pueden destacar los estudios de Pérez y Gallardo, 2002; Pérez Gallardo y de la Peña, 2005; Moya, 2008, con la exploración de temas específicos centrados en los métodos de investigación (Palenzuela, Moreno y Arroyo, 2004), así como el análisis de la producción científica de los investigadores españoles en las revistas de contabilidad (Amat Salas et al., 2005) y las citas bibliográficas (Amat Salas et at., 1998). Y en los EE.UU, en el que se ofrece nuevas perspectivas sobre la contabilidad de gestión (Baiman, 1982; Brown y Gardner, 1985; Brown et al.,1987; Birnberg et al., 1990; Atkinson et al., 1997; Shields, 1997; Ittner y Larcker, 2001; Hesford et al., 2007).

Este conocimiento sobre la evolución y estado actual de la contabilidad de gestión puede proporcionar información importante sobre sus niveles de desarrollo, limitaciones y perspectivas de futuro. Así, el objetivo del presente trabajo es identificar y analizar los temas y métodos de investigación aplicada en los estudios de contabilidad de gestión en España, Brasil y de Lengua Española.

La justificación del estudio no se centra únicamente en la percepción de que el desarrollo de la ciencia depende de los estudios capaces de producir un conjunto de conocimientos sustantivos, sino también a la incorporación de nuevos investigadores dedicados al análisis y la reflexión crítica sobre la producción científica generada. Además los estudios de esta naturaleza, sirven como una herramienta de diagnóstico para investigar el fortalecimiento de la contabilidad de gestión, y detectar la estructura y evolución de este campo del conocimiento científico. 


\section{PLATAFORMA TEÓRICA - CONTABILIDAD DE GESTIÓN}

La contabilidad de gestión empezó a reconocerse como una ciencia social a partir de los años 60 del siglo pasado (Ryan, Scapens y Theobald, 2002). En general, el énfasis radicaba en el empirismo y el positivismo (Zimmerman, 1979; Watts y Zimmerman, 1979), que desemboca en el crecimiento de los estudios de casos y de campo en Europa (Panozzo, 1997, Drury y Taylor, 1994; Drury y Taylor, 2005) y la progresión evidente de la disciplina académica de la contabilidad de gestión.

Durante las etapas iniciales de su ejecución, la contabilidad de gestión se vinculó estrechamente a las teorías económicas, pero este ámbito se ha ampliado constantemente con la inclusión de las ciencias sociales, sociología, estudios de psicología y de organización, y el análisis de las matemáticas y la filosofía. Estos desarrollos han ayudado al fortalecimiento interdisciplinario y la expansión de su alcance, y sobre todo ha dado credibilidad académica y profesional (Baldvinsdottir; Norreklit y Mitchell, 2010).

Para el Institute of Management Accountants, contabilidad de gestión es una profesión que implica ayudar al proceso de toma de decisiones, en la elaboración de la planificación y los sistemas de gestión del rendimiento, y en la prestación de habilidades relacionadas con la información financiera y de control, lo que ayuda a los administradores en la formulación y aplicación de la estrategia en la organización (IMA, 2008).

\subsection{Cuestiones clave en estudios de contabilidad de gestión.}

Basado en el trabajo de Brown y Gardner (1985), Brown et al. (1987), Shields (1997), Gallardo, Pérez y Peña (2005), Schekaiban y Ripoll (2005), Hesford et al. (2007), Lunkes, Ripoll y Rosa (2010), los artículos han sido clasificados por líneas de investigación o temas.

Como punto de partida para la clasificación de los elementos utilizados en la contabilidad de gestión, se ha utilizado la división tradicional, método aceptado en la literatura, entre los "costes" y "planificación y control", además de temas específicos, denominados "otros". La división de los temas principales en las subdivisiones tuvo en cuenta los estudios de Ripoll y Schekaiban (2005) y el de Hesford et al. (2007).

La línea de investigación en Costes se divide 4 áreas. Contabilidad de costes, en donde se agrupan los problemas relacionados con la asignación de los costes, opciones o sistemas de cálculo de costes, costes de la subactividad e identificación de factores de coste y $\mathrm{ABC}$, entre otros. El área Gestión de costes analiza los cambios en el coste-volumen-beneficios (CVB), utilizados en la toma de decisiones. El área tres comprende Estudios y aplicaciones prácticas de coste, incorpora estudios históricos de costes, evolución de los de sistemas de costes en el tiempo y análisis en diferentes países. Y, por último, esta línea de investigación en costes, incluye la gestión estratégica de costes, en donde se contemplan la gestión por procesos y la gestión de las por actividades (Activity-Based Management).

La línea de planificación y el control está subdividida en temas específicos para facilitar 
la clasificación de los estudios, que están relacionados con: presupuesto, presupuesto de capital, la medición y evaluación del desempeño, control organizacional y el control internacional.

La presupuestación incluye estudios actuales relacionados con los diferentes procesos de presupuesto (empresa, continuo, de base cero, para las actividades y Beyond Budgeting), ciclo presupuestario, el desarrollo la ejecución y el control presupuestario y las ventajas y limitaciones. El presupuesto de capital involucra cuestiones tales como las decisiones de inversión, los métodos de análisis de la inversión (VAN, TIR, recuperación de la inversión, la tasa de distribución de retorno y el descuento de flujos de efectivo) así como el coste de oportunidad. La medición y evaluación del desempeño incluye estudios relacionados con los sistemas de medición del desempeño y medidas de incentivo, que incluye las herramientas para la evaluación estratégica, el Cuadro de Mando o Tableau de Bord, Cuadro de Mando Integral o Balanced Scorecard y el Valor Económico Añadido. Hay una sub-categoría, que incluye los estudios de controles y aplicaciones en diferentes áreas de la organización, como el desarrollo de los controles internos y el trabajo en las áreas de responsabilidad (dentro de la organización). Por último, el control internacional que cubre estudios relacionados con el control en diferentes países y culturas (entre organizaciones).

La tercera y última línea, Otros Temas, agrupa nueve categorías, que son: sistema de información contable (SIC), evaluación comparativa (benchmarking), calidad (TQM), Just-InTime (JIT), Teoría de la Limitaciones (TOC), coste objetivo, la enseñanza de la contabilidad de gestión, gestión estratégica, precios de transferencia y el precio de venta, estudios sobre la contabilidad de gestión y análisis de los indicadores financieros. Los temas como la evaluación comparativa (benchmarking), calidad (TQM), Just-In-Time (JIT), Teoría de Restricciones (TOC), precio de transferencia y el precio de venta son fáciles de entender y clasificar. El SIC se caracteriza por estudios centrados en los sistemas de información como ERP. El tema de la educación de gestión consiste en estudios relacionados con la disciplina de la contabilidad de gestión y estrategias de enseñanza.

La gestión estratégica implica el trabajo relacionado con la alineación entre la estrategia de la organización y la gestión de sistemas de control operativos que permitan a la organización la consecución de su estrategia. El tema estudios sobre la investigación del CG recoge trabajos sobre su evolución y el estado del arte, en donde se analizan los métodos de investigación y los enfoques teóricos utilizados. Por último, el tema de análisis financiero incorpora el cálculo y uso de los indicadores financieros en la toma de decisiones para evaluar y comparar los resultados.

\subsection{Estudios de la Producción Científica en Contabilidad de Gestión}

Los estudios sobre la contabilidad de gestión pueden tener diferentes perspectivas, el enfoque en este estudio se centra en la producción científica. Para hacer la revisión de la literatura se han consultado dos bases de datos internacionales.

La primera está compuesta por la base de datos SciELO (Scientific Electronic Library Online), donde han sido encontrados cinco estudios sobre la producción científica en contabilidad de gestión, y la segunda base de datos utilizada ha sido ISI, donde se encontraron nueve estudios. En el Cuadro 1 se muestran los autores y una breve descripción de cada trabajo. 


\begin{tabular}{|c|c|}
\hline Autores & Breve Descripción de los Estudios \\
\hline $\begin{array}{l}\text { Carvalho et al., } \\
\quad 2010\end{array}$ & $\begin{array}{l}\text { Se analiza las aportaciones de las teorías del ciclo de vida de la organización en la } \\
\text { investigación en contabilidad de gestión. Se desarrolló un estudio bibliométrico con la base } \\
\text { de datos ProQuest }{ }^{\circledR} \text { que analizaba otros } 22 \text { estudios. }\end{array}$ \\
\hline $\begin{array}{l}\text { Hesford e Potter, } \\
\quad 2010\end{array}$ & Análisis de los artículos publicados sobre Contabilidad en la revista Cornell Hospitalidad. \\
\hline $\begin{array}{l}\text { Nascimento, } \\
\text { Junqueira e } \\
\text { Martins, } 2010\end{array}$ & $\begin{array}{l}\text { Se llevó a cabo un estudio bibliométrico y un análisis de contenido del Congreso de la } \\
\text { Asociación Nacional de Programas de Postgrado en Ciencias Contables (ANPCONT } \\
\text { Brasil), } 2007 \text { y 2008. Congresos de Contabilidad y Control de la Universidad de São Paulo } \\
\text { (Brasil).USP/Brasil) y Encontros Nacionais da Associação Nacional de Pós-Graduação } \\
\text { Pesquisa em Administração (ANPAD/Brasil) en los años } 2005 \text { a 2008, detectando un tota } \\
\text { de } 287 \text { trabajos. }\end{array}$ \\
\hline $\begin{array}{l}\text { Maloni, Carter y } \\
\quad \text { Carr, } 2009\end{array}$ & $\begin{array}{l}\text { Estudio que evaluó la investigación en el campo de la medición del desempeño y la a } \\
\text { concentración de autores en revistas, en un período de dieciséis años. Se analizaron } 1.796 \\
\text { artículos en cinco publicaciones desde } 1992 \text { hasta } 2007 \text {. }\end{array}$ \\
\hline $\begin{array}{l}\text { Mendonça, } \\
\text { Riccio e Sakata, } \\
2009\end{array}$ & $\begin{array}{l}\text { Analiza de la evolución temporal de la ciencia Contable en Brasil y la relación entre los } \\
\text { enfoques normativos y positivistas. Se analizaron los registros de ENANPADs en el período } \\
\text { comprendido entre } 1996 \text { y } 2005 \text {. }\end{array}$ \\
\hline $\begin{array}{l}\text { Alcouffe, } \\
\text { Berlande e } \\
\text { Levant, } 2008\end{array}$ & $\begin{array}{l}\text { El estudio tiene como objetivo encontrar el proceso de construcción de redes de } \\
\text { investigadores contables sobre todo, en la Contabilidad de costes por actividades (ABC). }\end{array}$ \\
\hline $\begin{array}{l}\text { Beuren, } \\
\text { Schlindwein e } \\
\text { Pasqual, } 2007\end{array}$ & $\begin{array}{l}\text { El artículo analiza el perfil de la investigación en Contabilidad de los artículos publicados } \\
\text { en el Congreso EnANPAD y el Congreso de Control y Contabilidad de la USP (Brasil) en } \\
\text { el período } 2001 \text { a } 2006 \text {. }\end{array}$ \\
\hline $\begin{array}{l}\text { Cardoso, Pereira } \\
\text { e Guerreiro, } \\
\quad 2007\end{array}$ & $\begin{array}{l}\text { Es un estudio diagnóstico, del perfil de la investigación de los costes centrado en el área de } \\
\text { contabilidad y gestión dentro del Congreso EnANPAD (Brasil), de } 1998 \text { a } 2003 \text {. El análisis } \\
\text { consistió inicialmente en una revisión de } 170 \text { artículos. Se seleccionaron } 32 \text { artículos de } \\
\text { costes. }\end{array}$ \\
\hline $\begin{array}{l}\text { Groot e Garcia- } \\
\text { Valderrama, } \\
\quad 2006\end{array}$ & $\begin{array}{l}\text { Este trabajo es un estudio bibliometrico de } 169 \text { grupos de investigación en economía } \\
\text { econometría y administración de empresas en los Países Bajos. Las evaluaciones se llevan } \\
\text { a cabo en dos periodos en } 1995 \text { y en } 2001 \text {. }\end{array}$ \\
\hline $\begin{array}{l}\text { Valacich, et al., } \\
\quad 2006\end{array}$ & $\begin{array}{l}\text { Se examina la proporción de artículos publicados en revistas de las mejores escuelas de } \\
\text { negocios entre los años } 1994 \text { a } 2003 \text {. }\end{array}$ \\
\hline $\begin{array}{l}\text { Tahai e Meyer, } \\
1999\end{array}$ & $\begin{array}{l}\text { El estudio analizó } 23.637 \text { referencias de revistas científicas citadas en } 1.275 \text { artículos } \\
\text { publicados en } 17 \text { revistas de gestión durante el período comprendido entre } 1993 \text { y } 1994 \text {. }\end{array}$ \\
\hline Engwall, 1998 & $\begin{array}{l}\text { El estudio examina los autores franceses que publican en revistas internacionales de } \\
\text { contabilidad de gestión, su origen geográfico y la orientación de estos autores. Se analizaron } \\
\text { las quince mejores revistas de gestión durante el período comprendido entre } 1981 \text { y } 1992 .\end{array}$ \\
\hline White, 1996 & $\begin{array}{l}\text { El estudio ha identificado en diferentes revistas, } 125 \text { medidas relacionadas con la estrategia } \\
\text { a través del análisis de temas relacionados con la contabilidad de gestión. }\end{array}$ \\
\hline $\begin{array}{l}\text { Kirkpatrick e } \\
\text { Locke, } 1992\end{array}$ & $\begin{array}{l}\text { Este estudio presenta una metodología para evaluar la concesión de becas. Se utilizaron } \\
\text { tres fuentes de información (artículos, citas y revisión por pares) fueron evaluados } 2.229 \\
\text { profesores de } 32 \text { escuelas de negocios en los Países Bajos. }\end{array}$ \\
\hline
\end{tabular}

Cuadro 1: Estudios de la producción científica en contabilidad de gestión

Como se aprecia en el Cuadro 1, resulta evidente que existen pocos estudios sobre los 
temas y métodos de contabilidad de gestión.

\section{METODOLOGÍA DE LA INVESTIGACIÓN}

En este epígrafe se presentan los procedimientos metodológicos utilizados para construir el marco teórico y el proceso de selección y clasificación de los artículos. Esta encuesta sigue la estructura de la investigación llevada a cabo por Baldvinsdottir; Norreklit y Mitchell (2010) para la contabilidad de gestión, y se desarrolla en tres etapas.

\subsection{Proceso estructurado para la creación del marco teórico}

El proceso estructurado para la selección y análisis de referencias se divide en tres fases: la elección de la base de datos, la selección de artículos y el análisis sistémico (Rosa, Ensslin y Ensslin, 2009). La primera fase justifica la elección de la base de datos, la segunda obtiene una cartera de artículos basados en un proceso estructurado, y la última, proporciona un análisis sistémico de los elementos de la cartera de artículos. Sobre la base de esta estructura fueron seleccionados para el análisis sistémico 14 artículos que estaban alineados con el tema de la literatura, en producción científica, en contabilidad de gestión.

En primer lugar, se realizó una consulta en la base de datos Scientific Electronic Library Online - SciELO y el International Statistical Institute - ISI, utilizando las palabras clave "Management Accounting", "Contabilidad de Gestión" y "Contabilidade Gerencial". Fueron encontrados 6.969 artículos en la base de datos ISI. Con el fin de detectar solo artículos y temas relacionados con la gestión, se aplicados estos dos filtros. De esta forma la muestra obtenida constó de 412 artículos. Con los mismos filtros de la base Scielo fueron seleccionados 472 artículos.

A partir de esta muestra inicial se analizaron los resúmenes para seleccionar los artículos relacionados en la literatura científica en contabilidad de gestión. De esta selección se detectaron 5 artículos de la base de datos Scielo y 9 trabajos de la base ISI, que constituyen la base teórica de nuestro trabajo.

\subsection{Procedimiento para la selección de los artículos}

Este epígrafe tiene como objetivo analizar las publicaciones en revistas editadas en España, en Brasil y en publicaciones de Lengua Española en materia de contabilidad. Para seleccionar los artículos se consideró un período de 10 años (2001-2010).

Para la selección de revistas en España se considera el término "Contabilidad"o "contable" y su inclusión en la base de datos IN-RECS Índice (impacto de las revistas españolas de ciencias sociales, desarrollado por la Universidad de Granada, siendo utilizado para la selección de las revistas del factor de impacto acumulado (citas por artículo hasta 0,01). La elección de IN RECS ha sido debida a su amplio uso en España, y la imposibilidad de utilización de otra base de datos, ya que España tiene una sola revista con índice de impacto en materia contable (por ejemplo, en ISI - International Statistical Institute ou JCR - Journal Citation Reports). La 
Revista Española de Financiación y Contabilidad es la única indexada como JCR.

En este periodo, han sido seleccionadas siete revistas españolas, de acuerdo a la Tabla 1. Siendo la Revista Iberoamericana de Contabilidad de Gestión la única publicación exclusivamente en contabilidad de gestión y por lo tanto todos los artículos obtenidos fueron incluidos en la muestra. Se seleccionaron 421 artículos relacionados con la contabilidad de gestión. Esta selección se realizó mediante la lectura del resumen y, el análisis, del artículo en su totalidad. No se incluyeron artículos relacionados con notas de investigación, entrevistas, reseñas de libros, tesis y editoriales.

Tabla 1: Resultados de las revistas seleccionadas

\begin{tabular}{|c|c|c|}
\hline Revistas & $\begin{array}{l}\text { Total de artículos } \\
\text { (2001 a 2010) }\end{array}$ & $\begin{array}{c}\text { Artículos Seleccionados } \\
(2001 \text { a } 2010)\end{array}$ \\
\hline Revista Española de Financiación y Contabilidad - REFC & 332 & $40(12 \%)$ \\
\hline Revista de Contabilidad - RC & 117 & $15(13 \%)$ \\
\hline Revista de Contabilidad y Tributación - RCT & 764 & $18(2,5 \%)$ \\
\hline $\begin{array}{c}\text { Partida Doble Revista de Contabilidad, Auditoría y } \\
\text { Empresa - RCAE }\end{array}$ & 921 & $89(9,5 \%)$ \\
\hline Técnica Contable - RTC & 612 & $44(7 \%)$ \\
\hline $\begin{array}{l}\text { Revista Iberoamericana de Contabilidad de Gestión - } \\
\text { RICG }\end{array}$ & $125^{*}$ & $125(100 \%)$ \\
\hline \multirow[t]{2}{*}{ Harvard-Deusto Finanzas \& Contabilidad - RHDFC } & $449 * *$ & $90(20 \%)$ \\
\hline & 3.320 & $421(12 \%)$ \\
\hline
\end{tabular}

* Disponible a partir de 2003. *** Publicación de una edición de la revista en 2001.

En Brasil se han considerado los términos "contábil" y "contabilidade" y su inclusión en la base de datos "Qualis" CAPES (Coordinación de Perfeccionamiento de la Educación Superior), con la categorización A1, A2, B1, B2 y B3. Estas, son consideradas las mejores revistas, sirviendo, además para evaluar los programas de posgrado en el país. El área de conocimiento "contabilidad" no tiene ninguna revista en las categorías A1 y A2, ni revistas indexadas por el ISI y JCR.

Se seleccionaron seis revistas brasileñas, que aparecen en la Tabla 2. La muestra está compuesta de 245 artículos. Los resultados muestran que del total de las publicaciones en contabilidad, el 30,5\% son artículos en contabilidad de gestión. Datos que están por encima del $12 \%$ de los españoles y $28 \%$ de los anglosajones.

Tabla 2: Resultados de las revistas brasileñas seleccionadas

\begin{tabular}{|c|c|c|c|}
\hline Revistas & $\begin{array}{c}\text { Ponderación } \\
\text { CAPES }\end{array}$ & $\begin{array}{c}\text { Total de } \\
\text { artículos } \\
(2001 \text { a 2010) }\end{array}$ & $\begin{array}{c}\text { Artículos } \\
\text { Seleccionados } \\
(2001 \text { a 2010) }\end{array}$ \\
\hline Revista de Contabilidade \& Finanças - RCF & B1 & 210 & $63(30 \%)$ \\
\hline Revista de Contabilidade Vista \& Revista - RCVR & B3 & 210 & $80(38 \%)$ \\
\hline Revista Universo Contábil - RUC & B3 & $164 * *$ & $53(32 \%)$ \\
\hline Revista Contemporânea de Contabilidade - RCC & B3 & $85^{*}$ & $40(47 \%)$ \\
\hline Revista de Contabilidade e Organizações - RCO & B3 & $73 * * *$ & $19(26 \%)$ \\
\hline Revista de Educação e Pesquisa em Contabilidade - REPC & B3 & $60 * * *$ & $10(17 \%)$ \\
\hline Total & - & 802 & $245(30,5 \%)$ \\
\hline
\end{tabular}

* Disponible a partir de 2004, **2005 y *** 2007. 
Para la selección de las revistas de Lengua Española se utilizó la base de datos Latindex, que es un sistema de información sobre las revistas de investigación científica, técnicoprofesionales y de divulgación científica y cultural que se editan en los países de América Latina, el Caribe, España y Portugal, (búsqueda de catálogo) para hacer la selección de las revistas de lengua española y se seleccionaron aquellas correspondientes con el área de conocimiento de "Ciencias Sociales", que contenía 2.874 revistas. Desde esta área, se seleccionó el ámbito de la contabilidad, que está formado por 31 revistas. De esta selección inicial se excluyeron las revistas publicadas en lengua portuguesa (15 revistas), y da España (2 revistas), y las que no ofrecen elementos suficientes para su consulta por medios electrónicos ( 9 revistas).

Así se seleccionaron cinco revistas de lengua española, que aparecen en la Tabla 3. La muestra está compuesta de 52 artículos.

Tabla 3: Resultados de las revistas de lengua española

\begin{tabular}{c|c|c}
\hline Revistas seleccionadas & $\begin{array}{c}\text { Total de artículos } \\
(\mathbf{2 0 0 1} \text { a 2010) }\end{array}$ & $\begin{array}{c}\text { Artículos seleccionados } \\
(\mathbf{2 0 0 1} \text { a 2010) }\end{array}$ \\
\hline RCN-Contabilidad y Negocios (Perú) & $52^{* *}$ & $8(15,5 \%)$ \\
\hline REGD-Economía, Gestión y Desarrollo (Colombia) & $86^{*}$ & $4(7,5 \%)$ \\
\hline RSE-Semestre Económico (Colombia) & $86^{* * *}$ & $3(5,5 \%)$ \\
\hline RACF-Actualidad Contable Faces (Venezuela) & $134^{*}$ & $27(52 \%)$ \\
\hline RCA-Contaduría y Administración (México) & 193 & $10(19,5 \%)$ \\
\hline Total & 551 & $52(23 \%)$ \\
\hline
\end{tabular}

* Disponible a partir de 2003. ** Disponible a partir de 2006. *** Disponible a partir de 2004.

Los resultados muestran que en España no ha habido un crecimiento en las publicaciones en contabilidad de gestión, incluso con la creación de la revista exclusiva RICG. Tal y como se muestra en el gráfico 1, el mejor año fue 2003, con 63 artículos, año de la aparición de la RICG, observándose una disminución a partir de ese año.

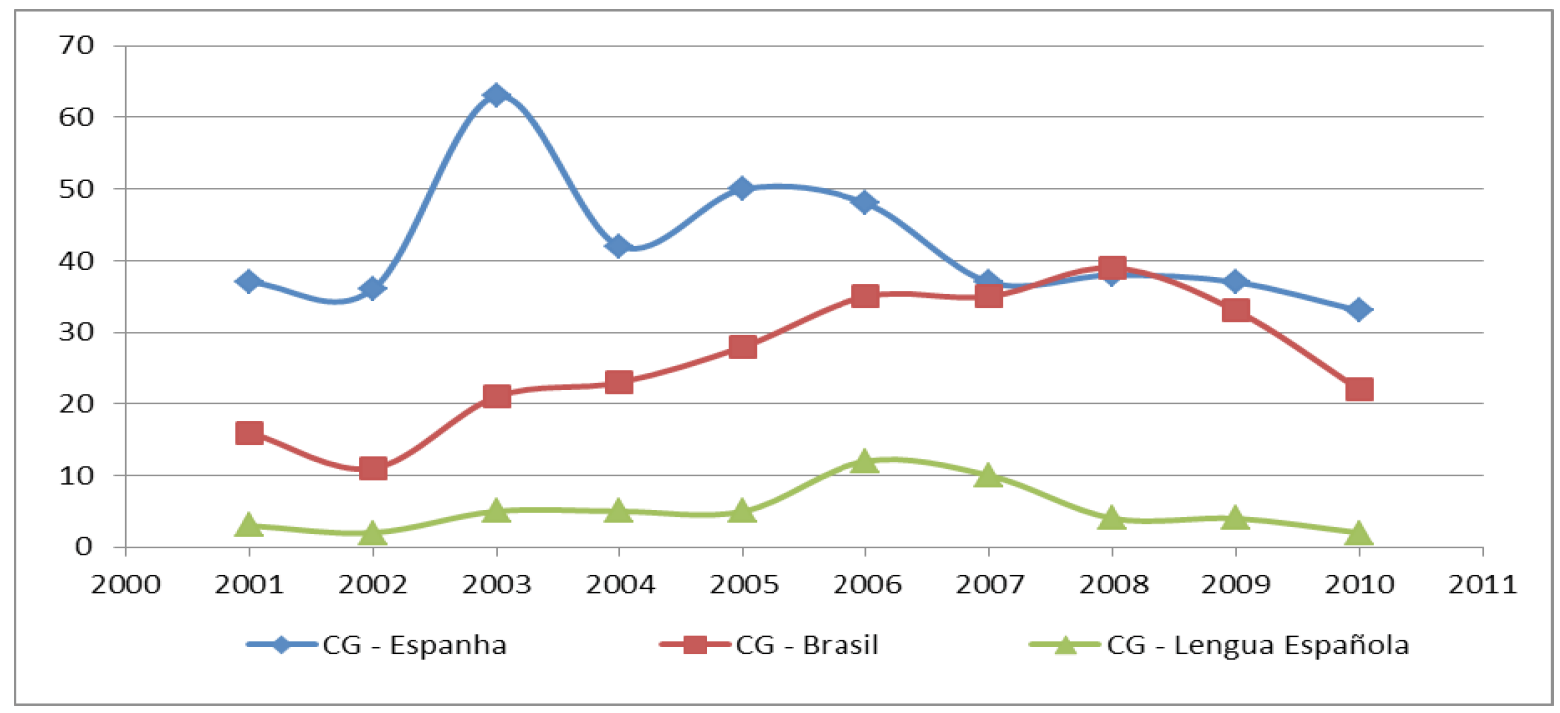

Gráfico 1: Número de artículos publicados en CG en España, Brasil y de Lengua Española

Debido a la aparición de la RICG en 2003, en donde se publicaron 20 artículos, fue el período de mayor incremento en las publicaciones en contabilidad de gestión. La publicación 
anual media es de aproximadamente 42 artículos. Esto demuestra que a diferencia de otros países, por ejemplo, EE.UU., donde hubo un aumento en términos absolutos y un descenso en términos relativos (Hesford et al., 2007), las publicaciones en España, en contabilidad de gestión, han sufrido una disminución en los últimos años en términos relativos y absolutos.

En Brasil, el área de la contabilidad en general está creciendo, con nuevas revistas, desde el inicio del período que estamos analizando, ya que al inicio del periodo solamente había dos revistas. En el gráfico 1, se observa que el número de publicaciones ha experimentado un rápido crecimiento, en el periodo inicial, con una disminución en los dos últimos años en términos absolutos, y una disminución en términos relativos en los últimos cuatro años. Con un promedio de 25 publicaciones al año, número que es aún bajo comparado con España.

Los resultados de las revistas de lengua española muestran que aproximadamente el $23 \%$ de los artículos publicados durante el periodo están relacionados con la contabilidad de gestión. En el gráfico 1, se observa que el número de publicaciones ha experimentado un crecimiento en 2006 y 2007, en el periodo inicial, con una disminución en los tres últimos años.

\subsection{Procedimientos para la clasificación en temas e métodos de investigación}

Para ordenar los artículos en temas fueron utilizados como referencia los trabajos de Brown y Gardner (1985), Brown et al. (1987), Shields (1997), Gallardo Pérez y Peña (2005), Schekaiban y Ripoll (2005), Hesford et al. (2007), Lunkes, Ripoll y Rosa (2010), tal como indicamos en el epígrafe 2.1 .

En cuanto a la clasificación de los artículos de acuerdo con los métodos de investigación se utilizó el trabajo de Birnberg et al. (1990) y Hesford et al. (2007), que dividen los métodos en nueve categorías, que se describen en el Cuadro 2.

\begin{tabular}{|c|c|}
\hline Métodos & Definición \\
\hline Analítica & $\begin{array}{l}\text { Se trata de la evaluación y el estudio detallado de la información disponible en un intento de } \\
\text { explicar el contexto de un fenómeno. }\end{array}$ \\
\hline $\begin{array}{l}\text { Archivo/ } \\
\text { documental }\end{array}$ & Implica el uso de la información que no ha tenido un tratamiento científico. \\
\hline Caso & $\begin{array}{l}\text { Se trata de la investigación de los fenómenos, incluyendo a las personas, procedimientos y } \\
\text { estructuras en una sola organización. }\end{array}$ \\
\hline Experimental & $\begin{array}{l}\text { Implica el tratamiento de la información en un intento de establecer causa-efecto de las } \\
\text { variables investigadas. }\end{array}$ \\
\hline Campo & $\begin{array}{l}\text { Se trata de la investigación de los fenómenos, incluyendo a las personas, procedimientos y } \\
\text { estructuras en dos o más organizaciones. }\end{array}$ \\
\hline Framework & Implica el desarrollo de un nuevo concepto, nuevas perspectivas. \\
\hline Revisión & Básicamente consiste en la síntesis y la revisión de la literatura ya conocida. \\
\hline $\begin{array}{l}\text { Survey } \\
\text { (encuesta) }\end{array}$ & $\begin{array}{l}\text { Se trata de la investigación de un grupo de personas, a menudo llevado a cabo a través de un } \\
\text { cuestionario. }\end{array}$ \\
\hline Otros & No encaja en ninguno de los otros métodos. \\
\hline
\end{tabular}

Cuadro 2: Clasificación y definición de métodos de investigación 
Con el objetivo de clasificar los artículos de la muestra se llevó a cabo la identificación del tema y el método de investigación aplicado.

\section{RESULTADOS DE LA INVESTIGACIÓN}

Para poder concluir los resultados del análisis, los artículos han sido clasificados por temas y métodos de investigación, tal como se describe en la metodología de la investigación.

\subsection{Clasificación de los Artículos en los temas}

La identificación de los temas nos permite comprender las principales líneas de investigación en España, Brasil y de Lengua Española. En España y de lengua española el publicaciones se centraron en otros asuntos de contabilidad de gestión, se relacionados con planificación y control y costes. En Brasil el tema más destacado fue el de planificación y control, seguido de otros temas y costes, como se muestra en la Tabla 4.

Tabla 4: Resultados de la clasificación de los temas

\begin{tabular}{|c|c|c|c|c|}
\hline Temas & 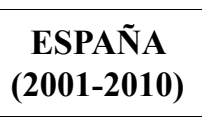 & $\begin{array}{c}\text { BRASIL } \\
(2001-2010)\end{array}$ & $\begin{array}{c}\text { LENGUA } \\
\text { ESPAÑOLA } \\
(2001-2010)\end{array}$ & Total \\
\hline \multicolumn{5}{|l|}{ Costes } \\
\hline Contabilidad de costes & $43(10,5 \%)$ & $25(10 \%)$ & $7(13,5 \%)$ & $75(10,5 \%)$ \\
\hline Gestión de Costes & $7(1,5 \%)$ & $28(11,5 \%)$ & $3(5,5 \%)$ & $38(5 \%)$ \\
\hline Estudios y prácticas de costes & $27(6,5 \%)$ & $6(2,5 \%)$ & $7(13,5 \%)$ & $40(5,5 \%)$ \\
\hline Gestión estratégica de costes & $14(3,5 \%)$ & $\mathrm{N} / \mathrm{A}$ & $1(2 \%)$ & $15(2 \%)$ \\
\hline Múltiples & $17(4 \%)$ & N/A & - & $17(2,5 \%)$ \\
\hline Sub-total & $108(26 \%)$ & $59(24 \%)$ & $18(34,5 \%)$ & $185(25,5 \%)$ \\
\hline \multicolumn{5}{|l|}{ Planificación y control } \\
\hline Presupuesto & $34(8 \%)$ & $6(2,5 \%)$ & $1(2 \%)$ & $41(5,5 \%)$ \\
\hline Presupuesto de capital & $5(1 \%)$ & $24(9,5 \%)$ & $3(6 \%)$ & $32(4,5 \%)$ \\
\hline Medición y evaluación del desempeño & $55(13 \%)$ & $31(12,5 \%)$ & $6(11,5 \%)$ & $92(13 \%)$ \\
\hline Control organizacional & $21(5 \%)$ & $39(16 \%)$ & $4(7,5)$ & $64(9 \%)$ \\
\hline Control internacional & $3(1 \%)$ & $11(4,5 \%)$ & - & $14(2 \%)$ \\
\hline Múltiples & $9(2 \%)$ & $2(1 \%)$ & - & $11(1,5 \%)$ \\
\hline Sub-total & $127(30 \%)$ & $113(46 \%)$ & $14(27 \%)$ & $254(35,5 \%)$ \\
\hline \multicolumn{5}{|l|}{ Otros } \\
\hline Sistema de información contable - SIC & $26(6 \%)$ & $23(9,5 \%)$ & $1(2 \%)$ & $50(7 \%)$ \\
\hline Benchmarking & $4(1 \%)$ & $1(0,5 \%)$ & - & $5(0,5 \%)$ \\
\hline Calidad (TQM) & $25(6 \%)$ & $3(1,5 \%)$ & $5(9,5 \%)$ & $33(4,5 \%)$ \\
\hline Just-in-time (JIT) e TOC & $2(0,5 \%)$ & $7(3 \%)$ & $3(5,5 \%)$ & $12(1,5 \%)$ \\
\hline Educación en gestión & $4(1 \%)$ & $19(7,5 \%)$ & $\mathrm{N} / \mathrm{A}$ & $23(3,5 \%)$ \\
\hline Gestión estratégica & $12(3 \%)$ & $18(7 \%)$ & $3(6 \%)$ & $33(4,5 \%)$ \\
\hline $\begin{array}{l}\text { Precios de transferencia y Precios de } \\
\text { venta }\end{array}$ & $17(4 \%)$ & $2(1 \%)$ & $1(2 \%)$ & $20(3 \%)$ \\
\hline Estudios en CG & $28(6,5 \%)$ & N/A & N/A & $28(4 \%)$ \\
\hline Análisis Financiero & $31(7,5 \%)$ & N/A & $\mathrm{N} / \mathrm{A}$ & $31(4,5 \%)$ \\
\hline Múltiples & $37(8,5 \%)$ & 0,0 & $7(13,5 \%)$ & $44(6 \%)$ \\
\hline Sub-total & $186(44 \%)$ & $73(30 \%)$ & $20(38,5 \%)$ & $279(39 \%)$ \\
\hline Total & $421(100 \%)$ & $245(100 \%)$ & $52(100 \%)$ & $718(100 \%)$ \\
\hline
\end{tabular}

N/A: No aplicable. 
Si observamos los datos que se desprenden de las revistas anglosajonas, apreciamos que la planificación y control es el tema mayormente discutido, seguido por el de coste y otros temas de contabilidad de gestión (Hersford et al., 2007), mientras que en España, Brasil y de lengua española, las investigaciones se centran, generalmente, en temas más periféricos.

Los tópicos destacados en revistas de España y de lengua española son medición y evaluación del desempeño y la contabilidad de costes. En Brasil y en las revistas anglosajonas, entre los temas más importantes de investigación, destacan el control organizacional, la medición y evaluación del desempeño.

Entre los elementos con pocas publicaciones en contabilidad en España son "just in time", teoría de las restricciones, el presupuesto de capital, el control organizativo y de gestión en la educación. En Brasil, los artículos raramente encontrados en las publicaciones son el estudio y la práctica de costes, presupuesto, calidad y precio de venta y transferencia. Y de lengua española son los tópicos control organizacional y benchmarking.

En general, los resultados muestran que en España la contabilidad de gestión se encuentra más consolidada, a diferencia de Brasil que presenta un importante crecimiento (tanto de revistas como de investigadores), debido a una incipiente red organizada, que dará sus frutos en los años venideros con la aparición de investigadores de referencia.

Un paso importante hacia la consolidación del área de conocimiento de contabilidad de gestión es el desarrollo de un núcleo de temas centrales basado en teorías aceptadas (Hopwood, 2007 e 2008; Modell, 2010; Vaivio e Sirén, 2010; Merchant, 2010; Baldvinsdottir; Mitchell e Norreklit, 2010). Tal vez otro paso clave que contribuya a la promoción y mejora de la calidad del trabajo en sus aspectos descriptivos y normativos es la consolidación de su propia metodología que se aplicará en la investigación sobre la contabilidad de gestión (Zimmerman, 2001; Ittner e Lacker, 2001 e 2002; Baldvinsdottir; Mitchell y Norreklit, 2010).

\subsection{Métodos de Investigación}

En la identificación y el análisis de los métodos de la investigación aplicada es importante entender cómo el área lleva a cabo su investigación. En la tabla 4, se muestra como en España la revisión de la literatura y los métodos de estudio de casos son los temas más predominantes. En Brasil, el foco está en el estudio de caso y framework. Y en revistas de lengua española, el foco está en la revisión de la literatura y estudios de casos.

Si comparamos los resultados de España, Brasil y de lengua española con la doctrina anglosajona, donde se destacan los métodos de framework y estudio analítico, parece que la manera de investigar difiere entre países, por ejemplo en el caso español e de lengua española, los investigadores se centran más en trabajos de corte descriptivo. 
Tabla 5: Resultados de los métodos de investigación

\begin{tabular}{c|c|c|c|c}
\hline Métodos de Investigación & $\begin{array}{c}\text { ESPAÑA } \\
(\mathbf{2 0 0 1 - 2 0 1 0})\end{array}$ & $\begin{array}{c}\text { BRASIL } \\
(\mathbf{2 0 0 1 - 2 0 1 0 )}\end{array}$ & $\begin{array}{c}\text { LENGUA ESPAÑOLA } \\
(\mathbf{2 0 0 1 - 2 0 1 0})\end{array}$ & Total \\
\hline Analítica & $33(8 \%)$ & $10(4 \%)$ & - & $43(6 \%)$ \\
\hline Archivo/ documental & $7(2 \%)$ & $10(4 \%)$ & - & $17(2,5 \%)$ \\
\hline Caso & $120(28,5 \%)$ & $54(22 \%)$ & $12(23 \%)$ & $186(26 \%)$ \\
\hline Experimental & $4(1 \%)$ & $8(3,5 \%)$ & - & $12(1,5 \%)$ \\
\hline Campo & $58(13,5 \%)$ & $15(6 \%)$ & $10(19,5 \%)$ & $83(11,5 \%)$ \\
\hline Framework & $9(2 \%)$ & $66(27 \%)$ & - & $75(10,5 \%)$ \\
\hline Revisión & $137(32,5 \%)$ & $43(17,5 \%)$ & $24(46 \%)$ & $204(28,5 \%)$ \\
\hline Survey (encuesta) & $52(12 \%)$ & $36(14,5 \%)$ & $6(11,5 \%)$ & $94(13 \%)$ \\
\hline Otros & $1(0,5 \%)$ & $3(1,5 \%)$ & - & $4(0,5 \%)$ \\
\hline Total & $421(100 \%)$ & $245(100 \%)$ & $52(100 \%)$ & $718(100 \%)$ \\
\hline
\end{tabular}

Las investigaciones anglosajonas plantean nuevas propuestas metodológicas, mientras que en España y de lengua española aún existe una tendencia a discutir temas consolidados y aplicarlos a la práctica, mientras que en Brasil la preocupación es contribuir a los programas de investigación empírica y teórica.

Entre los métodos utilizados en la investigación relativamente escasos en contabilidad de gestión en España y de lengua española son los estudios con la aplicación del método experimental, de archivo y documental. En Brasil, la investigación experimental, análisis y archivo documental son muy poco utilizados. Y de lengua española son los estudios con la aplicación del método experimental, de archivo y documental, analítica y framework.

Los resultados muestran que existe un gran potencial para la investigación en estudios de campo en Brasil, España y en países latino-americanos de lengua española dentro del marco conceptual.

\section{CONCLUSIONES Y RECOMENDACIONES}

Desde el objetivo descrito, analizamos 461 publicaciones en España, 245 de Brasil y 52 de lengua española, relacionados con la contabilidad de gestión, que fueron ordenados por líneas de investigación (costes, planificación y control y otro) y por metodologías de investigación. Los resultados de nuestro trabajo ponen de manifiesto que la contabilidad de gestión no ocupa un lugar destacado en las publicaciones en revistas españolas, brasileñas y de lengua española. Esto explica en parte por qué muchos países en esta área de investigación detectan la carencia de formación, y la falta de centros especializados en el ámbito de la contabilidad de gestión, y la incorporación de redes consolidadas de autores de diferentes países y zonas.

Para Pérez Gallardo y Peña (2005), la explicación a esto puede ser: (i) ausencia de bases de datos, como las existentes en otras vertientes tales como la contabilidad financiera 
y (ii) el número limitado de revistas especializadas en contabilidad de gestión. En nuestro estudio, sólo una revista tiene un enfoque en esta materia. En este mismo sentido, Ittner y Larcker (2001) mencionan la dificultad de la obtención de datos, en comparación otras áreas del conocimiento donde existen bases de datos muy potentes y muy bien comercializadas. A pesar de que Zimmerman (2001) niega las acusaciones afirmando que el fracaso de la contabilidad de gestión, entre otras razones es la falta de creatividad de sus investigadores.

Se cree que la consolidación en el ámbito de la contabilidad de gestión debe ocurrir debido a una serie de medidas que deben tomarse en cuenta por los investigadores Brasileños, Españoles y de otros países latino-americanos, tales como: (i) la realización de investigaciones e intercambio internacional para promover la expansión del espectro del marco teórico y metodológico para la contabilidad de gestión y construir o reforzar las redes sociales de los investigadores, (ii) la ampliación del marco de la investigación y estudios de casos, y (iii) el fortalecimiento de canales permanentes de comunicación científica en el área (por ejemplo, revistas y creación de bases de datos).

En general, los investigadores en contabilidad de gestión en Brasil, España y de lengua española tienen una fuerte tendencia a la búsqueda de temas periféricos (línea de investigación "otros"), en comparación con los costes y la planificación y control, mediante el estudio de métodos de investigación del caso y revisión. Si se comparan estos resultados con el trabajo de Hesford et al. (2007), las publicaciones son limitadas en temas de contabilidad de gestión - gestión de costes y control estratégico-, con aplicaciones de métodos de investigación experimental y analítico. Esto demuestra que hay un gran potencial para los investigadores brasileños, españoles y de países latino-americanos en futuros estudios con la aplicación de estos métodos y temas en el ámbito de la contabilidad de gestión.

En este sentido, Merchant (2010), señala que las escuelas de negocios de EE.UU. tienen un enfoque restringido, lo cual proporciona una gran oportunidad para las universidades en Europa y otras partes del mundo como América del Sur, con el fin de tomar una posición de liderazgo en muchas áreas de investigación importantes, tales como la contabilidad de gestión. Pero esa oportunidad se perderá si las universidades tratan de emular el modelo americano de las escuelas de negocios.

Para Merchant (2010) la escuela americana tiene una serie de restricciones con respecto a determinados métodos de investigación, sobre todo, los centrados en investigación de corte cualitativo. Sus enfoques son más centrados en teorías económicas a diferencia de otros países en donde se utilizan múltiples teorías (de la organización, contingente etc.). En este sentido, los investigadores españoles y brasileños tienen un papel importante por su formación más completa fuertemente arraigada en las disciplinas de la sociología y la psicología, la aplicación de enfoques multidisciplinarios a las demandas de esta área del conocimiento.

Entre las recomendaciones para futuros trabajos, creemos que existe la posibilidad de ampliar la investigación para incluir otras bases de datos como ISI, SciELO, Dialnet, Latindex etc., lo que aumentará la posibilidad de la inclusión de revistas anglosajonas, y de los países de habla española y portugués, entre otras posibilidades. 


\section{REFERENCIAS}

ALCOUFFE, S.; BERLANDEE, N.; LEVANT, Y. Actor-networks and the diffusion of management accounting innovations: A comparative study. Management Accounting Research. Vol.19 (1), 2008, p. 1-17.

AMAT SALAS, O., BLAKE, J. GOWTHORPE, C., SOBREVIAS, E.O. Analisis de autores, citas y revistas de contabilidad em España. Revista Española de Financiación y Contabilidad, Vol. XXVI (96), 1998, p.821-850.

ATKINSON, A.; R. BALAKRISHNAN; P. BOOTH; J. COTE; T. GROOT; T. MALMI; H. ROBERTS; E. ULIANA, A.; WU, A. New Directions in Management Accounting Research. Journal of Management Accounting Research, 9, 1997, p. 79-108.

ATKINSON, A. A. et al. Contabilidade gerencial. São Paulo: Atlas, 2000.

ANTHONY. R. N.; GOVINDARAJAN, V. Sistemas de controle gerencial. São Paulo: Atlas, 2001.

BAIMAN, S. Agency research in managerial accounting: a survey. Journal of Accounting Literature, Vol.1, 1982. p.154-213.

BALDVINSDOTTIR, G.; MITCHELL, F.; NORREKLIT, H.. Issues in the relationship between theory and practice in management accounting. Management Accounting Research. Vol.21 (2), 2010, p. 79-82.

BEUREN, I. M.; SCHLINDWEIN, A. C.; PASQUAL, D. L. Abordagem da controladoria em trabalhos publicados no EnANPAD e no Congresso USP de controladoria e contabilidade de 2001 a 2006. Revista Contabilidade \& Finanças. Vol.18(45), 2007, p. 22-37.

BIRNBERG, J., SHIELDS, M., YOUNG, S. The case for multiple methods in empirical management accounting research (with an illustration from budget setting). Journal of Management Accounting Research, Vol. 2 (Fall), 1990, p.33-66.

BROMWICH, M. The case for strategic management accounting: the role of accounting information for strategy in competitive markets. Accounting, Organizations and Society, 15(1), 1990, p.27-46.

BROWN, L. D. GARDNER, J. C. Applying citation analysis to evaluate the research contributions of accounting faculty and doctoral programs. The Accounting Review. Vol. (45), 1985 , p. 262-277.

BROWN, L. D. GARDNER, J. C. Using citation analysis to assess the impact of journals and articles on contemporary accounting research (CAR). Journal of Accounting Research. Vol. (46), 1985, p. 84-109.

BROWN, L. D., GARDNER, J.C. VASARHELYI, M.A.An analysis of the research contributions of Accounting, Organizations and Society, 1976-1984. Accounting, Organizations and Society. Vol. (12), 1987, p. 193-204.

CARDOSO, R. L.; PEREIRA, C. A.; GUERREIRO, R. Perfil das pesquisas em contabilidade de custos apresentadas no EnANPAD no período de 1998 a 2003. Revista de Administração Contemporânea. Vol. 11(3), 2007, p. 177-198.

CARVALHO, K. L.; SARAIVA JÚNIOR, A. F.; FREZATTI, F.; COSTA, R. P. A contribuição das teorias do ciclo de vida organizacional para a pesquisa em contabilidade gerencial. RAM Revista de Administração da Mackenzie (Online) 11(4), 2010, p. 98-130. 
CHAPMAN, C.S.; HOPWOOD, A.G.; SHIELDS, M.D. Handbook of Management Accounting Research. Elsevier, 2007.

COOPER, D. J. Tidiness, muddle, and things: commonalities and divergencies in two approaches to management accounting research. Accounting, Organizations and Society, 8(2/3), 1983, p.269-286.

COOPER, R. Does your company need a new cost system?. Journal of Cost Management, $1(1), 1987$, p.45-49.

COVALESKI , M. A.; DIRSMITH, M. W.; SAMUEL, S. Managerial accounting research: the contributions of organizational and sociological theories. Journal of Management Accounting Research, 8, 1996, p.1-35.

DENT, J. F. Strategy, organization, and control: some possibilities for accounting research. Accounting, Organizations and Society, 15(1/2), 1990, p.3-25.

EKHOLM, B., WALLIN, J. Is the annual budget really dead? European Accounting Review. Vol. 9. 2000, p. 519-539.

ENGWALL, L. Research note: Asterix in Disneyland. Management scholars from France on the world stage. Organizations Studies. Vol. 19 (5), 1998, p. 863-881.

DRURY, C.; TAYLES, M. Product Costing in UK manufacturing Organizations. The European Accounting Review. Vol.3 (3), 1994, p. 443-469.

DRURY, C.; TAYLES, M. Explicating the Design of Overhead Absorption Procedures in UK Organizations. British Accounting Review. Vol.37 (3), 2005, p. 47-84, .

ENGWALL, L. Research note: Asterix in Disneyland. Management scholars from France on the world stage. Organizations Studies. Vol. 19 (5), 1998, p. 863-881.

ESCOBAR PEREZ, B.; LOBO GALLARDO, A. Implicaciones teóricas y metodológicas de la evolución de la investigación en contabilidad de gestión. Revista Española de Financiación y Contabilidad, Vol. XXXI (111), 2002, p. 245-286.

GONZÁLES, C. L. Producción Científico en Contabilidad: El caso de España (1992-2002). Revista Española de Financiación y Contabilidad, Vol. XXXIV, 5, Abril - Junio, 2005, p.363-393.

GROOT, T.; GARCIA-VALDERRAMA, T. Research quality and efficiency - An analysis of assessments and management issues in Dutch economics and business research programs. Research Policy. Vol.35 (9), 2006, p. 1362-1376.

HESFORD, J. W.; POTTER, G. Accounting Research in the Cornell Quarterly: A Review with Suggestions for Future Research. Cornell Hospitality Quarterly. Vol. 51 (4), 2010, p. 502512 .

HESFORD, S.R.; et al. Management accounting: a bibliographic study. In: CHAPMAN, Christopher; HOPWOOD, Anthony G.; SHIELDS, Michael (Org.). Handbook of management accounting research. Amsterdam: Elsevier, 2007.

HIROMOTO, T. Another hidden edge: Japanese management accounting. Harvard Business Review, 66(4), 1988, p.22-25.

HOPWOOD, A. G. If only there were simple solutions, but there aren't: some relections on Zimmerman's critique of empirical management accounting research. European Accounting Research, v. 11 (4), 2002, p. 777-785. 
HOPWOOD, A.G. Whither accounting research? The Accounting Review. Vol. 82, 2007, p.1365-1374.

HOPWOOD, A.G. Management accounting research in a changing world. Journal of Management Accounting Research 20, 2008, p.3-13.

IMA - Institute of Management Accountants. Definition of Management Accounting. Institute of Management Accountants, IMA, 2008.

ITTNER, C. D.; LARCKER, D. F. Assessing empirical research in managerial accounting: a value-based management perspective. Journal of Accounting and Economics, v. 32, 2001, p. 349-410.

. Empirical managerial accounting research: are we just describing management consulting practice? European Accounting Research, Vol. 11 (4), 2002, p. 787-794.

JOHNSON, H. T.; KAPLAN, R. S. Relevance lost: the rise and fall of management accounting. Boston, MA: Harvard Business School Press, 1987.

KAPLAN, R. S. Measuring manufacturing performance: a new challenge for management accounting research. The Accounting Review, 58(4), 1983, p.686-705.

.; NORTON, D. The balanced scorecard: measures that drive performance. Harvard Business Review, 70(1), 1992, p.71-79.

. Organização orientada para a estratégia. Rio de Janeiro: Campus, 2001.

. Estratégia em Ação - Balanced Scorecard, 7ª. Ed. Rio de Janeiro: Campus, 1997.

KIRKPATRICK, A.S.; LOCKE, E.A. The Development of Measures of Faculty Scholarship. Group \& Organization Management. Vol. 17 (1), 1992, p. 5-23.

LUFT, J.; SHIELD, M. D. Mapping management accounting: graphics and guidelines for theory-consistent empirical research? Accounting, Organizations and Society, Vol. 28, 2003, p. 169-249.

Zimmerman's contentious conjectures: describing the present and prescribing the future of empirical management accounting research. European Accounting Review, Vol. 11 (4), 2002, p. 795-805.

LUKKA, K.; MOURITSEN, J. Homogeneity or heterogeneity of research in management accounting? European Accounting Review, Vol.11 (4), 2002, p. 805-811.

LUKKA, K. The roles and effects of paradigms in accounting research. Management Accounting Research. Vol. 21, 2010. p. 110-115.

LUNKES, R.J.; RIPOLL FELIU, V.M.; ROSA, F.S. Redes Sociais e Internacionalização Da Contabilidade Gerencial: Um Estudo em Publicações de Lengua Espanhola. Congresso de Contabilidade e Auditoria, Porto (Portugal), 2011, p.1-15.

MACINTOSH, N. B.; SCAPENS, R. W. Structuration theory in management accounting. Accounting, Organizations and Society, 15(5), 1990, p.455-477.

MALMI, T.; GRANLUND, M. In Search of Management Accounting Theory. The European Accounting Review. Vol. 18 (3), 2009. p. 597-620.

MALONI, M. J.; CARTER, C. R.; CARR, A. S. Assessing logistics maturation through author concentration. International Journal of Physical Distribution \& Logistics Management. 
Vol. 39 (3-4), 2009, p. 250-268.

MENDONÇA NETO, O. R.; RICCIO, E. L.; SAKATA, M. C. G. Dez anos de pesquisa contábil no Brasil: análise dos trabalhos apresentados nos ENANPADs de 1996 a 2005. Revista Administração de Empresas.Vol. 49(1), 2009, p. 62-73.

MERCHANT, K.A. Paradigms in accounting research: A view from North America. Management Accounting Research. Vol. 21 (2), 2010, p. 116-120.

MODELL, S. Bridging the paradigm divide in management accounting research: The role of mixed methods approaches. Management Accounting Research. Vol.21, 2010 p. 124-129.

MOYA, S.¿Quién Publica en las Revistas Españolas de Contabilidad? Análisis bibliométrico del periodo 1996-2005. Revista Española de Financiación y Contabilidad, Vol. XXXIV (138), 2008, p. 353-374.

NASCIMENTO, A. R.; JUNQUEIRA, E.; MARTINS, G. A. Pesquisa acadêmica em contabilidade gerencial no Brasil: análise e reflexões sobre teorias, metodologias e paradigmas. Revista de Administração Contemporânea. Vol. 14(6), 2010, p. 1113-1133.

PALANZUELA, V.A.; MORENO, B.P.; ARROYO, A.S. Verificación Empírica y Método del caso: Revisión de algunas experiencias en Contabilidad de Gestión a la Luz de su Metodología. Revista Española de Financiación y Contabilidad. Vol. 33 (121), 2004, p. 349-377.

PANOZZO, F. The making of the Good Academic Accountant. Accounting Organizations and Society. Vol.22 (5), 1997, p. 447-480.

PEREZ, B.E.; GALLARDO, A L. Implicaciones teóricas y metodológicas de la evolución de la investigación en contabilidad de gestión. Revista Española de Financiación y Contabilidad, Vol. XXXI (111), 2002, p.245-286.

PEREZ, B. E., GALLARDO, A. L., PEÑA, C.R. La investigación empírica en contabilidad de gestión en España: análisis de las publicaciones españolas. Revista Española de Financiación y Contabilidad, Vol. XXXIV, No.124, Enero - Marzo, 2005, p.183-210.

ROSA, F. S., ENSSLIN, S. R., ENSSLIN, L. Evidenciação Ambiental: Processo Estruturado de Revisão de Literatura sobre Avaliação de Desempenho da Evidenciação Ambiental. Sociedade, Contabilidade e Gestão, Vol. 4, 2009, p. 4-8.

RYAN, R.; SCAPENS, R.W.; THEOBALD, M. Research Method \& Methodology in Finance Accounting. Thomson, 2002.

SCHEKAIBAN, N.; RIPOLL, V.M. Estudio Comparativo sobre Investigación en Contabilidad de Gestión: Espana-México, 2005.

SHANK, J.; GOVINDARAJAN, V. Strategic cost management: the new tool for competitive advantage. New York, NY: Free Press, 1993.

SHIELDS, M. D. Research in management accounting by North Americans in the 1990s. Journal of Management Accounting Research, Vol. 5, 1997, p. 3-61.

SIEGEL, G., KULESZA, C. From Statement Preparer to Decision-Support Specialist: The Coming Changes in Management Accounting Education. Management Accounting. Vol.32, 1996, p. 34-45.

SIMONS, R. Levers of Control - How Managers Use Innovative Control Systems to Drive Strategic Renewal. Boston: Harvard Business, 1995. 
TAHAI, A.; MEYER, M.J. A revealed preference study of management journals' direct influences. Strategic Management Journal. Vol.20 (3), 1999, p. 279-296.

WATTS, R.; ZIMMERMAN, J. The Demand dor and Supply of Accounting Theories. The Market for Escuses. The Accounting Review. Vol. 59, 1979, p. 273-305.

WHITE, G.P. A survey and taxonomy of strategy-related performance measures for manufacturing. International Journal of Physical Distribution \& Logistics Management. Vol. 16 (3), 1996, p. 42-68.

YOUNG, S. M.; SELTO, F. H. New manufacturing practices and cost management: a review of the literature and directions for future research. Journal of Accounting Literature, 10, 1991, p.265-298.

VAIVIO, J.; SIRÉN, A. Insights into method triangulation and "paradigms" in interpretive management accounting research. Management Accounting Research Vol.21, 2010, p. 130141.

VALACICH, J.S.; FULLER, M.A.; SCHNEIDER, C.; DENNIS, A.R. Issues and opinions Publication opportunities in premier business outlets: How level is the playing field? Information System Research. Vol. 17 (2), 2006, p. 107-125.

ZIMMERMAN, J.L. The Cost and Benefits and Cost Allocations. The Accounting Review. Vol. 54, 1979, p. 504-521.

ZIMMERMAN, J. L. Conjectures regarding empirical managerial accounting research. Journal of Accounting and Economics, Vol. 32, 2001, p. 411-427. 


\section{ENDEREÇO DOS AUTORES:}

\section{Rogério João Lunkes}

Universidade Federal de Santa Catarina, Centro Sócio-Econômico, Departamento de Ciências Contábeis,

Campus Universitário, Trindade

88040-900 - Florianópolis, SC - Brasil

\section{Vicente Mateo Ripoll Feliu}

Universitat Valencia.

Avgda. dels Tarongers, sn

46071 - Valencia, - Espanha

\section{Fabricia Silva da Rosa}

Universidade Federal de Santa Catarina, Centro Sócio-Econômico, Departamento de Ciências Contábeis,

Campus Universitário, Trindade

88040-900 - Florianópolis, SC - Brasil 